\title{
An Overview of the Historical Development of Antimalaria Drug Resistance
}

\section{Olumohunle Folake*, Iorzua Doosuur, Idris Surayyah, Baba Aishatu N and Gangbe Hope}

Department of Biological Science, School of life science, Federal university of technology, Minna, Nigeria

*Corresponding author: Olumohunle Folake, Department of Biological Science, School of life science, Federal university of technology, Minna, Nigeria, E-mail: flakylizzy@gmail.com

Received: June 19, 2018; Accepted: June 30, 2018; Published: July 7, 2018

\section{Abstract}

Antimalarial drugs are key tools for the control and elimination of malaria. Recent decreases in the global malaria burden are likely due, in part, to the deployment of artemisinin-based combination therapies. Therefore, the emergence and potential spread of artemisinin-resistant parasites and changes in sensitivities to artemisinin partner drugs have raised concerns. Research activities include clinical studies to track the efficacies of antimalarial drugs, ex vivo/in vitro assays to measure drug susceptibilities of parasite isolates, and characterization of resistance-mediating parasite polymorphisms. These efforts offer an increasingly comprehensive assessment of the efficacies of antimalarial therapies, and enable us to predict the emergence of drug resistance and to guide local antimalarial drug policies. Here we briefly review worldwide antimalarial drug resistance concerns.

Keywords: Historical; Antimalarial; Drugs; Therapies

\section{Introduction}

Malaria remains an important public health concern in countries where transmission occurs regularly, as well as in areas where transmission has been largely controlled or eliminated. In Africa, for instance, people can be exposed to as many as 300 infective bites per year (in rare cases, even as much as 1000 infective bites per year), and during peak transmission, as many as five infective bites per night and this leads to high prevalence of malaria especially in Africa [1]. The disease varies widely in epidemiology and clinical manifestation in different parts of the world. This variability is the result of factors such as the species of malaria parasites that occur in a given area, their susceptibility to commonly used or available antimalarial drugs, the distribution and efficiency of mosquito vectors, climate and other environmental conditions and the behaviour and level of acquired immunity of the exposed human populations. In 1976 WHO defined drug resistance as the ability of the parasite strain to survive or multiply despite the administration or absorption of a drug given in doses equal to or higher than those usually recommended but within the tolerance of the subject [2]. The definition was later modified to include the sentence, "the form of the drug active against the parasite must be able to gain access of the parasite or the infected erythrocyte for the duration of the time necessary for its normal action'. [2]. This addition took into account a new

Citation: Folake O, Doosuur I, Surayyah I, et al. An Overview of the Historical Development of Antimalaria Drug Resistance. J Curr Chem Pharm Sci. 2018; 8(1):112.

(C) 2018 Trade Science Inc. 
www.tsijournals.com | July-2018

development in the understanding of human metabolism of sulphonamide. As the pharmacokinetics of antimalarial medicines varies widely among individuals, the definition of the resistance is enhanced if the concentration profile of the active drug concerned is also taken into consideration. For example, in the case of a prodrug, which is not active in the ingested form and require chemical conversion by metabolic process to become pharmacologically active [3]. Antimalarial drug resistance has emerged as one of the greatest challenges facing malaria control today. Drug resistance has been implicated in the spread of malaria to new areas and re-emergence of malaria in areas where the disease had been eradicated. Drug resistance has also played a significant role in the occurrence and severity of epidemics in some parts of the world. Population movement has introduced resistant parasites to areas previously free of drug resistance.

\section{History of Antimalarial-Drug Resistance}

Drugs have been used to treat and prevent malaria for many centuries. So far, no preventative vaccination against malaria exists, and its control depends heavily upon antimalarial drugs that kill parasites inside the human body. Malaria has been noted for more than 4,000 years. Back from the cinchona tree, which contained an array of alkaloids with antimalarial properties, appeared in Western therapeutics in the 17th century. One of the alkaloids, quinine, was isolated in 1820 and became the drug of choice for treating malaria until World War II. In ancient China, India, the Middle East, Greece, and Rome, malaria and its possible treatments were documented. Ancient Chinese used a treatment based on artemisinin (documented $168 \mathrm{BC}$ ), the active ingredient in some high-end drugs nowadays. Treatments with Quinine are known to the western world since the early 17th century [4]. In British colonies, tonic water (which contains large amounts of Quinine) was mixed with gin and became a popular drink. The discovery of Chloroquine (CQ) in the 1930s revolutionized malaria treatments. CQ was the most widely-used drug from the early 1950s to until the 1990s. After about ten years of use, mutations within P. falciparum that conferred resistance to CQ arose independently in Columbia and Thailand [4]. Since then CQ-resistant mutations have been spreading quickly through most endemic areas. CQ clears out resistant parasites less efficiently from the human body than sensitive (non-resistant) parasites. Sulfadoxine-pyrimethamine (SP), a combination of two drugs, was used to replaced CQ. However, resistance to SP evolved rapidly and now occurs at high frequency in major malarious regions [4]. Currently, alternative drugs (e.g., artemisinin-based combination therapies) are available and others continue to be developed. However, higher production costs limit their widespread application in major endemic areas. The evolution of resistance against affordable drugs incurs an enormous societal cost for fighting the spread of the disease [4]. Development of resistance requires a high grade of parasitemia, coupled with low or inadequate drug levels. Most cases of resistance has emerge out of South-East Asia region. This region is known for low transmission and low immunity that lead to high parasetemia; it also has a long history of indiscriminate use of different antimalarial drugs [5]. Areas with very high transmission such as Africa, appear to be less susceptible to the emergence of drug resistance. In these areas, infections are acquired throughout life, resulting in partial immunity, that in turns control the infection, usually at levels below than those that cause symptoms. Asymptomatic infection and often non-availability of drugs in these areas mean that these patients do not receive antimalarial drugs, and hence the chances development of resistance are low [6]. Immunity act by non-selecting eliminating blood-stage parasites, including the rare resistant de novo mutants, and also improve cure rate, even with failing drug, thereby reducing the relative transmission advantage of resistant parasites.

\section{Mechanisms of Antimalarial Resistance}


www.tsijournals.com | July-2018

For some drugs, only a single point mutation is required to confer resistance, while for other drugs, multiple mutations appear to be required. Provided the mutations are not deleterious to the survival or reproduction of the parasite, drug pressure will remove susceptible parasites while resistant parasites survive. Single malaria isolates have been found to be made up of heterogeneous populations of parasites that can have widely varying drug response characteristics, from highly resistant to completely sensitive [7]. Similarly, within a geographical area, malaria infections demonstrate a range of drug susceptibility. Over time, resistance becomes established in the population and can be very stable, persisting long after specific drug pressure is removed. The biochemical mechanism of resistance has been well described for chloroquine, the antifolate combination drugs, and atovaquone.

\section{Resistance to Quinine}

Resistance to quinine, the oldest antimalarial drug, was reported first in Brazil and later in southeast Asia. Quinine resistance is associated with polymorphisms in several transporters, nmely:Polymorphisms in the Plasmodium falciparum multidrug resistance-1 (pfmdr1) and Plasmodium falciparum multidrug resistance protein-1 (Pfmrp1) are linked to decreased sensitivity to quinine. In addition, pfmdr1 gene amplification can also lead to quinine resistance [8]. Recent studies evaluating associations between polymorphisms in microsatellite, in vitro parasite sensitivity, and clinical responses to various drugs have been inconsistent, but these polymorphisms appear to have a modest impact on sensitivity of parasites to quinine, and possibly other drugs.

\section{Chloroquine resistance}

As the malaria parasite digests haemoglobin, large amounts of a toxic by-product are formed. The parasite polymerizes this by-product in its food vacuole, producing non-toxic haemozoin (malaria pigment). It is believed that resistance of $P$. falciparum to chloroquine is related to an increased capacity for the parasite to expel chloroquine at a rate that does not allow chloroquine to reach levels required for inhibition of haem polymerization [1]. This chloroquine efflux occurs at a rate of 40 to 50 times faster among resistant parasites than sensitive ones [10]. Further evidence supporting this mechanism is provided by the fact that chloroquine resistance can be reversed by drugs which interfere with this efflux system [6].

\section{Antifolate Combination Drugs}

Antifolate combination drugs, such as sulfadoxine + pyrimethamine, act through sequential and synergistic blockade of 2 key enzymes involved with folate synthesis. Pyrimethamine and related compounds inhibit the step mediated by dihydrofolate reductase (DHFR) while sulfones and sulphonamides inhibit the step mediated by dihydropteroate synthase (DHPS). Specific gene mutations encoding for resistance to both DHPS and DHFR have been identified. Specific combinations of these mutations have been associated with varying degrees of resistance to antifolate combination drugs [2].

\section{Atovaquone}

Atovaquone acts through inhibition of electron transport at the cytochrome $b c l$ complex [10]. Although resistance to atovaquone develops very rapidly when used alone, when combined with a second drug, such as proguanil (the combination used in MalaroneTM) or tetracycline, resistance develops more slowly. Resistance is conferred by single-point mutations in the cytochrome-b gene. 


\section{Resistance to artemisinin family drugs}

Since artemisinins play an indispensable role in current malaria therapies, artemisinin resistance has received wide recent attention. In the Cambodia- Thailand border region of southeast Asia, an epicenter of antimalarial drug resistance, declining efficacy of the artesunate/mefloquine combination was noted, and clinical resistance to artesunate, manifested as delayed clearance of parasitemia after therapy, but not generally as full-blown treatment failure. Very recently, using clinical and molecular data, the extent of artemisinin resistance has been delineated, with delayed clearance are common parts of Cambodia, Thailand, Myanmar, and Vietnam, but not in other areas of Asia or Africa [11]. In African parasites gene polymorphisms are common, including some mutations in the propeller domain, the specific mutations associated with artemisinin resistance in southeast Asia have not been seen. Parasites with introduced K13 mutations showed enhanced survival after a dihydroartemisinin pulse, confirming the role of these mutations in resistance. The transcriptomes of resistant parasites showed increased expression of unfolded protein response pathways and prolonged ring-stage development, offering insights into the mehanism of artemisinin resistance [11].

\section{Factors contributing to the spread of resistance}

Numerous factors contributing to the advent, spread, and intensification of drug resistance exist, although their relative contribution to resistance is unknown. Factors that have been associated with antimalarial drug resistance include such disparate issues as human behaviour (dealt with in detail elsewhere), vector and parasite biology, pharmacokinetics, and economics [12]. As mentioned previously, conditions leading to malaria treatment failure may also contribute to the development of resistance.

\section{Biological influences on resistance}

Based on data on the response of sensitive parasites to antimalarial drugs in vitro and the pharmacokinetic profiles of common antimalarial drugs, there is thought to always be a residuum of parasites that are able to survive treatment . Under normal circumstances, these parasites are removed by the immune system (non-specifically in the case of non-immune individuals). Factors that decrease the effectiveness of the immune system in clearing parasite residuum after treatment also appear to increase survivorship of parasites and facilitate development and intensification of resistance [13]. The nonspecific immune response of non-immune individuals is less effective at clearing parasite residuum than the specific immune response of semi-immune individuals. There is some evidence that certain combinations of drug-resistant parasites and vector species enhance transmission of drug resistance, while other combinations inhibit transmission of resistant parasites. In South-East Asia, two important vectors, Anopheles stephensi and A. dirus, appear to be more susceptible to drug-resistant malaria than to drug sensitive malaria [14]. Some malaria vectors may be somewhat refractory to drug-resistant malaria, which may partially explain the pockets of chloroquine sensitivity that remain in the world in spite of very similar human populations and drug pressure (e.g. Haiti). Many antimalarial drugs in current usage are closely related chemically and development of resistance to one can facilitate development of resistance to others. Chloroquine and amodiaquine are both 4aminoquinolines and cross-resistance between these two drugs is well known. Development of resistance to mefloquine may also lead to resistance to halofantrine and quinine. Antifolate combination drugs have similar action and widespread use of sulfadoxine/ pyrimethamine for the treatment of malaria may lead to increased parasitological resistance to other antifolate combination drugs. Development of high levels of SP resistance through continued accumulation of DHFR mutations may 
www.tsijournals.com | July-2018

compromise the useful lifespan of newer antifolate combination drugs such as chlorproguanil/dapsone (LapDap) even before they are brought into use. This increased risk of resistance due to SP use may even affect non-malarial pathogens; use of SP for treatment of malaria increased resistance to trimethoprim/ sufamethoxazole among respiratory pathogens. Parasites from new infections or recrudescent parasites from infections that did not fully clear will be exposed to drug blood levels that are high enough to exert selective pressure but are insufficient to provide prophylactic or suppressive protection Parasites reappearing during a period when blood levels were below the point required to clear pyrimethamine-resistant parasites, but still above that level required to clear pyrimethamine-sensitive parasites, were more likely to be pyrimethmaine-resistant than those reappearing after levels had dropped below the level required to clear pyrimethamine- sensitive parasites [15].

In areas of high malaria transmission, the probability of exposure of parasites to drug during this period of selective pressure is high. It is apparent that there are more genetically distinct clones per person in areas of more intense transmission than in areas of lower transmission. However, the interpretation of this and its implications for development of resistance has variously been described as resistance being more likely in low-transmission environments, high-transmission environments, or either low- or high- but not intermediate-transmission environments. This relationship between transmission intensity and parasite genetic structure is obviously complex and subject to other confounding/contributing factors [14].

\section{Genetic Signatures of Drug Resistance Evolution}

More insight for developing and maintaining an effective drug-deployment policy will come from advanced evolutionary genetic modeling. Policy components will require that the models incorporate key parameters that may determine how fast the resistance spreads. Important factors are geographically-specific variables (e.g., the transmission/migration rate and host immunity) and the evolutionary genetic structure of resistance (e.g., the number and fitness effects of mutated genes and their interactions). Currently only limited information regarding these parameters is available, which severely limits efforts to analyze and evaluate models [12]. One promising approach to obtain the necessary information is to reconstruct the actual events of drug-resistance evolution that recently occurred in various endemic areas. However, researchers have indirectly examined the past evolutionary dynamics of drug resistance by applying modern theories of population genetics to presentday samples of malarial parasite DNA.

\section{Programmatic influences on resistance}

Programmatic influences on development of antimalarial drug resistance include overall drug pressure, inadequate drug intake (poor compliance or inappropriate dosing regimens), pharmacokinetic and pharmacodynamic properties of the drug or drug combination, and drug interactions. Additionally, reliance on presumptive treatment can facilitate the development of antimalarial drug resistance. Studies have suggested that resistance rates are higher in urban and per urban areas than rural communities, where access to and use of drug is greater [16].

Concurrent treatment with other drugs can increase the likelihood of treatment failure and may contribute to development of drug resistance. Drug quality has also been implicated in ineffective treatment and possibly drug resistance. Either through poor manufacturing practices, intentional counterfeiting, or deterioration due to inadequate handling and storage, drugs may not contain sufficient quantities of the active ingredients. In an analysis of chloroquine and antibiotics available in Nigeria and Thailand, between 37\% and $40 \%$ of samples assayed had substandard content of active ingredients, mostly from poor manufacturing practices. Another study in Africa found chloroquine stored under realistic tropical conditions lost at least $10 \%$ of its activity in a little over a year [17]. 


\section{Conclusion}

In conclusion, because of the realities of health care infrastructure and the influence of the private sector, approaches to malaria therapy, especially in sub-Saharan Africa, will probably favour increased access to drugs (and, therefore, loss of control over how they are used) over restricted access (and, therefore, more control over how they are used). If this proves to be true, while only minor advances against antimalarial drug resistance can be expected, short-term reductions in malaria morbidity and mortality may be achieved.

\section{References}

1. Baird JK. Resistance to chloroquine unhinges vivax malaria therapeutics. Antimicrobial Agents Chemotherapy. 2011;55:1827-1830.

2. Yeung S, White L. Artemisinin resistance: current status and scenarios for containment. National Review of Microbiology. Journal Of Infectious Diseases. 2010;8:272-80.

3. Dondorp AM, Fanello CI, Hendriksen IC, Gomes E, Seni A, et al. Artesunate versus quinine in the treatment of severe falciparum malaria in African children (AQUAMAT): an open-label, randomised trial. Lancet. 2010;376:1647-1657.

4. Kim Y, Schneider KA. Evolution of Drug Resistance in Malaria Parasite Populations. Nature education knowledge. 2013;4:6.

5. Gosling RD, Okell L, Mosha J, Chandramohan D. The role of antimalarial treatment in the elimination of malaria. 2013;17:1617-1623.

6. Greenwood B. Anti-malarial drugs and the prevention of malaria in the population of malaria endemic areas. 2010;9:S2.

7. Koenderink JB, Kavishe RA, Rijpma SR, Russel FG. The ABCs of multidrug resistance in malaria. Trends Parasitology. 2010;26:440-446.

8. Conrad MD, LeClair N, Arinaitwe E, Wanzira H, Kakuru A,. Comparative impacts over 5 years of artemisininbased combination therapies on Plasmodium falciparum polymorphisms that modulate drug sensitivity in Ugandan Dondorp. Infectious Diseases. 2014;210:344-353.

9. Packard RM. History of medicine: The Origins of Antimalarial-Drug Resistance. New England Journal of Medicine. 2014;10:397-399.

10. Okumu FO, Moore SJ. Combining indoor residual spraying and insecticide- treated nets for malaria control in Africa: a review of possible outcomes and an outline of suggestions for the future. 2011;10:208.

11. Liwang C, Sungano M, Daouda N, Pradipsinh KR, Philip JR. Antimalarial Drug Resistance: Literature Review and Activities and Findings of the ICEMR Network. The American Journal of Tropical Medicine and Hygiene. 2015;93:57-68.

12. Sanchez CP, Dave A, Stein WD, Lanzer M. Transporters as mediators of drug resistance in Plasmodium falciparum. International Journal of Parasitology. 2010;40:1109-1118.

13. Tumwebaze P, Conrad MD, Walakira A, LeClair N, Byaruhanga O, et al. Four-year efficacy of RTS,S/AS01E and its interaction with malaria exposure. New England Journal of Medicine. 2013;368:1111-1120. 
www.tsijournals.com | July-2018

14. Bloome J, Okiring J, Kakuru A, Bigira V, Kapisi J, et al. Impact of antimalarial treatment and chemoprevention on the drug sensitivity of malaria parasites isolated from Ugandan children. Antimicrobial Agents Chemotherapy 2015;59:3018-3030.

15. Veiga MI, Ferreira PE, Jornhagen L, Malmberg M, Kone A, et al. Novel polymorphisms in Plasmodium falciparum ABC transporter genes are associated with major ACT antimalarial drug resistance. PLoS One. 2011;6:e20212.

16. Sharom FJ. The P-glycoprotein multidrug transporter. Essays Biochemistry. 2011;50:161-178.

17. Murray CJ, Rosenfeld LC, Lim SS, Andrews KG, Foreman KJ, et al. Global malaria mortality between 1980 and 2010: a systematic analysis. Lancet. 2012;379:413-431. 